\title{
The Smarter Sleep educational interventions: an initiative to reduce hypnotic prescribing in in-patient psychiatric care
}

\author{
Alastair Paterson, ${ }^{1,2}$ (1) Martina Khundakar, ${ }^{1,2}$ Anthony Young, ${ }^{1,3}$ Jonathan Ling, ${ }^{3}$ \\ Samantha Chakraborty, ${ }^{4}$ Adam Pattison Rathbone, ${ }^{2,5}$ Stuart Watson, ${ }^{2,6}$ Tim Donaldson, ${ }^{1}$ \\ Kirstie N. Anderson ${ }^{2,7}$
}

BJPsych Bulletin (2022) 46, 242-250, doi:10.1192/bjb.2021.41

\begin{abstract}
${ }^{1}$ Cumbria, Northumberland, Tyne and Wear NHS Foundation Trust, Newcastle upon Tyne, UK; ${ }^{2}$ Faculty of Medical Sciences, Newcastle University, Newcastle upon Tyne, UK; ${ }^{3}$ Faculty of Health Sciences and Wellbeing, University of Sunderland, UK; ${ }^{4}$ Department of General Practice, Monash University, Melbourne, Australia; ${ }^{5}$ Newcastle upon Tyne Hospitals NHS Foundation Trust, UK; ${ }^{6}$ Inpatient Services, Cumbria, Northumberland, Tyne and Wear NHS Foundation Trust, Newcastle upon Tyne, UK; ${ }^{7}$ Regional Sleep Disorders Service, Newcastle upon Tyne Hospitals NHS Foundation Trust, UK
\end{abstract}

Correspondence to Alastair Paterson (alastairpaterson1@icloud.com)

First received 28 Sep 2020, final revision 31 Mar 2021, accepted 9 Apr 2021

(C) The Author(s), 2021. Published by Cambridge University Press on behalf of the Royal College of Psychiatrists. This is an Open Access article, distributed under the terms of the Creative

Commons Attribution-NonCommercial-

ShareAlike licence (http://

creativecommons.org/licenses/by-nc$\mathrm{sa} / 4.0 /$ ), which permits non-

commercial re-use, distribution, and reproduction in any medium, provided the same Creative Commons licence is included and the original work is

properly cited. The written permission of

Cambridge University Press must be

obtained for commercial re-use.
Aims and method In-patients on mental health wards are commonly prescribed hypnotics for the long-term management of disturbed sleep. Specific sleep disorders remain underdiagnosed and effective behavioural interventions are underused. We developed a suite of three educational interventions (a video, poster and handbook) about sleep, sleep disorders, the safe prescribing of hypnotics and use of psychological strategies (sleep hygiene and cognitive-behavioural therapy for insomnia, CBTi) using co-design and multiprofessional stakeholder involvement. This controlled before-and-after study evaluated the effectiveness of these interventions across seven in-patient psychiatric wards, examining their impact on hypnotic prescribing rates and staff confidence scores (data collected by retrospective drug chart analysis and survey respectively).

Results A marked reduction was seen in the percentage of patients prescribed hypnotics on in-patient prescription charts ( $-24 \%)$, with a $41 \%$ reduction in the number of hypnotics administered per patient (mean reduction -1.142 administrations/patient).

Clinical implications These simple educational strategies about the causes and treatment of insomnia can reduce hypnotic prescribing rates and increase staff confidence in both the medical and psychological management of insomnia.

Keywords Sleep; hypnotics; benzodiazepines; deprescribing; implementation science.
Sleep and mental health are inextricably linked, with associations between abnormal sleep and depression, ${ }^{1}$ bipolar disorder, ${ }^{2}$ schizophrenia, ${ }^{3}$ autism spectrum disorders, ${ }^{4}$ attention-deficit hyperactivity disorder (ADHD) ${ }^{5}$ and relapse rates in addiction. ${ }^{6}$ Adequate duration and timing of sleep are essential for maintaining good physical and mental health. $^{7-9}$ Sleep disturbance has been associated with a two- to threefold increase in risk of psychiatric in-patient admission $^{10}$ and of suicide. ${ }^{11}$ The psychiatric in-patient setting in itself can be detrimental to sleep quality. ${ }^{12}$ As many as two-thirds of in-patients on psychiatric wards experience insomnia $^{13}$ and poor sleep is a predictor for psychiatric readmission. ${ }^{14}$

If left untreated, sleep disorders (Table 1) such as insomnia, narcolepsy, circadian rhythm disorders, parasomnias and restless legs syndrome can have deleterious effects on cardiovascular, metabolic and mental health, and some are linked to an increase in mortality ${ }^{15-17}$ 'Poor sleep' is not in its own right a diagnosis. ${ }^{18}$ This evidence-practice gap contributes to delays in diagnosis and treatment, ${ }^{19}$ an 


\begin{tabular}{|c|c|c|}
\hline Sleep disorder & Nature & Treatment \\
\hline Insomnia disorder & $\begin{array}{l}\text { Difficulty initiating and/or maintaining sleep, with daytime } \\
\text { impact }\end{array}$ & $\begin{array}{l}\text { Cognitive-behavioural therapy for insomnia (CBT-I) } \\
\text { Hypnotics }\end{array}$ \\
\hline Narcolepsy & $\begin{array}{l}\text { Neurological condition causing symptoms of excessive } \\
\text { sleepiness and sleep attacks }\end{array}$ & Refer to specialist \\
\hline Parasomnia & $\begin{array}{l}\text { Includes: sleepwalking, confusional arousals, night terrors, } \\
\text { rapid eye movement (REM) sleep behaviour disorder, sleep } \\
\text { paralysis, nightmare disorder }\end{array}$ & Refer to specialist \\
\hline $\begin{array}{l}\text { Circadian rhythm } \\
\text { disorders }\end{array}$ & $\begin{array}{l}\text { Disorder of the sleep-wake cycle (e.g. advanced/delayed } \\
\text { sleep phase syndrome, free running circadian rhythm } \\
\text { disorder) }\end{array}$ & $\begin{array}{l}\text { Sleep diaries/actigraphy for diagnosis and referral to } \\
\text { specialist }\end{array}$ \\
\hline $\begin{array}{l}\text { Restless leg } \\
\text { syndrome (RLS) }\end{array}$ & $\begin{array}{l}\text { Characterised by an unpleasant aching/creeping/crawling } \\
\text { sensation in the legs resulting in the irresistible urge to move; } \\
\text { often worse at night or at rest; may cause limb twitching } \\
\text { Precipitating factor for insomnia }\end{array}$ & $\begin{array}{l}\text { General practitioner management initially, with RLS-UK for } \\
\text { patient support } \\
\text { Lifestyle: avoid stimulants in the evening; quit smoking; } \\
\text { exercise; good sleep habits } \\
\text { Medicines: dopamine antagonists (pramipexole, ropinirole, } \\
\text { rotigotine) must be given with supervision given risk of } \\
\text { impulse control disorder). }\end{array}$ \\
\hline $\begin{array}{l}\text { Obstructive sleep } \\
\text { apnoea }\end{array}$ & $\begin{array}{l}\text { More prevalent in men over } 50 \text { who are overweight, } \\
\text { particularly in those with neck circumference }>43 \mathrm{~cm} \text { ( }>17 \\
\text { inches) } \\
\text { Alcohol and sedative medications also risk factors } \\
\text { Consider in those who have daytime sleepiness and a } \\
\text { witnessed snore }\end{array}$ & $\begin{array}{l}\text { Refer to specialist for sleep study for diagnosis } \\
\text { First line: continuous positive airway pressure (CPAP) } \\
\text { device } \\
\text { Second line (for mild to moderate disease only): mandibular } \\
\text { advancement device (MAD) }\end{array}$ \\
\hline
\end{tabular}

overprescribing of hypnotic medication, ${ }^{20}$ has a negative impact on recovery from psychiatric disorders and can cause misattribution of symptoms. ${ }^{21}$ Teaching on distinct sleep disorders and effective behavioural interventions for insomnia is generally lacking in UK undergraduate medical curricula. $^{22}$ In practice, an increase in prescribing rates, mortality and drug spend indicates a culture of inappropriate prescribing, suggesting that there is also a paucity of training about sleep disorders at graduate level. ${ }^{23}$

Hypnotics are widely prescribed for adult psychiatric in-patients suffering from poor sleep. ${ }^{24}$ They can be effective and necessary for acute insomnia, ${ }^{25}$ but if insomnia persists the benefits of hypnotics may wane while the risk of sideeffects increases. ${ }^{26}$ Hypnotics have the potential to worsen outcomes for sleep apnoea, anxiety, cognitive function and depression, ${ }^{27}$ and pose a significant risk of dependence and harm with long-term use. ${ }^{28}$ Hypnotics are commonly overprescribed: ${ }^{29}$ a 2015 survey of English general practice populations described inappropriate hypnotic prescribing as a public health problem, estimating that over a quarter of a million people take hypnotic medication beyond licensed timescales. ${ }^{30}$

Hypnotic medication should only be considered after appropriate assessment and after, or in combination with, psychological therapies such as cognitive-behavioural therapy for insomnia (CBTi). ${ }^{31}$ The deprescribing (cessation of inappropriate medication $)^{32}$ of hypnotic medication is considered problematic. ${ }^{33}$ A lack of institutional structures, inadequate resources, and the attitudes and practice of more senior clinicians have been cited as barriers to this process. ${ }^{34}$

At present, compliance with evidence-based care in sleep management is suboptimal. ${ }^{35}$ Increasingly, pharmacological agents are chosen over non-pharmacological treatments. ${ }^{36}$ Disturbances to sleep are multifactorial in nature. ${ }^{37}$ Although causal components can be tied to the patient's psychiatric diagnosis, underlying physical health condition or a distinct sleep disorder, there is a need for improved resourcing and greater awareness of assessment and treatment strategies to aid practitioners in managing insomnia ${ }^{38}$ and, more broadly, improving sleep on in-patient units. Barriers to implementation include: workload/time pressures, a lack of resources, a lack of authority to change practice, a workplace culture resistant to change and a lack of support from other staff members. ${ }^{39}$ There are modifiable behavioural factors that can markedly improve sleep. ${ }^{40}$ In in-patient settings, staff-related factors include reducing ward noise and overnight nursing observations, optimising the patient's bedroom for sleep and increasing staff awareness of sleep management. ${ }^{41}$ Patient-related factors include reducing caffeine, alcohol and nicotine consumption, ${ }^{42}$ setting a routine wake up time, introducing a wind-down period prior to attempting sleep and avoiding prolonged periods in bed while awake. ${ }^{43}$ Strategic factors include educational meetings, printed educational materials, local opinion leaders, revising professional roles and practice facilitation. Multifaceted, tailored strategies may be more effective at creating sustained change in practice. ${ }^{44}$

This study examines whether improving awareness of sleep, insomnia and behavioural sleep interventions among clinicians supports the deprescribing of hypnotics. The impact of a simple, online educational package on the prescribing patterns on in-patient mental health units was evaluated.

\section{Method}

\section{Design}

A co-design approach ${ }^{45}$ was employed to develop a package of educational interventions designed to be generalisable 


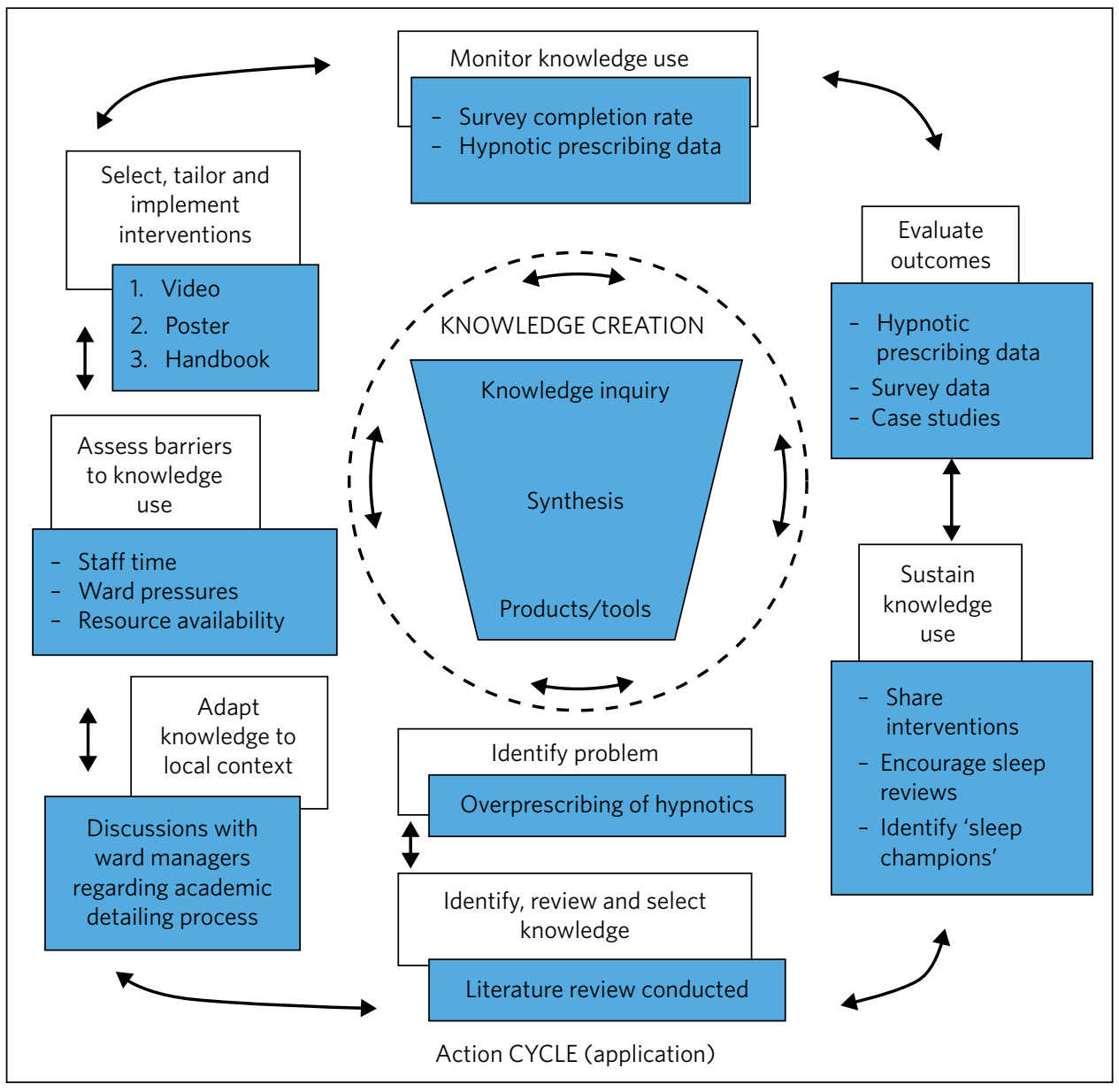

Fig. 1 The knowledge-to-action (KTA) framework (based on Graham et $\mathrm{al}^{46}$ ).

to practitioners across all care settings. The package, part of the Smarter Sleep educational materials (available from https://www.cntw.nhs.uk/resource-library/smarter-sleep/), comprised

- an overview video: a 9 min overview of normal sleep, specific sleep disorders, hypnotics and psychological therapies for insomnia

- a poster: to prompt nursing staff towards simple sleep interventions instead of hypnotic administration

- a handbook: a detailed review of sleep, causes of sleep disturbance and basic principles of CBTi.

These interventions were introduced to wards, and emails were sent each month as reminders that the interventions were active. Acute and rehabilitation wards for both male and female patients on one hospital site were eligible to participate. Wards on other sites within the trust (other than the control ward) were excluded.

\section{Development of the intervention}

The development, embedding and evaluation of these interventions can be mapped to the knowledge-to-action (KTA) framework ${ }^{46}$ (Fig. 1). This framework involves two phases - knowledge creation and the action cycle - which are interlinked and feed dynamically between each other.
Knowledge creation

Represented by a 'knowledge funnel', this consists of knowledge inquiry, synthesis and products/tools. This process encapsulates primary literature (knowledge inquiry) and secondary literature (synthesis), which are condensed down to produce 'third-generation knowledge' in the form of products/tools (decision aids, guidelines and pathways).

The action cycle

This part of the KTA framework represents the application of the knowledge creation process. It involves identifying the problem (the overprescribing of hypnotics) and then selecting the knowledge required for the intervention. We did this through literature review. ${ }^{47,48}$ Knowledge was then adapted to the local context through discussions with ward managers, and barriers to knowledge use were identified (time, ward pressures, resource availability). The interventions were tailored and implemented in the form of a video, poster and handbook, and knowledge use was monitored through the survey completion data and hypnotic prescribing rate data. Outcomes were evaluated using these data and case studies from implementation of the interventions. Knowledge use is being sustained through the embedding of interventions into everyday practice, and the hosting of the resources on the trust website (https://www.cntw.nhs. uk/smartersleep). 


\section{Hypnotic prescribing and administration rates}

A controlled before-and-after approach was taken to assess the impact of the interventions. Point prevalence data were collected via retrospective drug chart analysis over 2 full months (September 2019 and December 2019), with 2 months between for interventions to become embedded. These data consisted of a count of the number of active hypnotic prescriptions written on the drug charts of each ward during these periods. Data were also collected on the number of hypnotic administrations (i.e. individual doses) that took place on each pilot ward over this same periods. All doses in these time periods were counted, even if a patient was prescribed multiple hypnotic agents. These outcomes were relevant in evaluating the impact of the interventions on practice.

For example, seven doses administered against a prescription of 'zopiclone $7.5 \mathrm{mg}$ at night when required' would count as one active prescription and seven total administrations. Data were analysed using descriptive statistics.

\section{Pre- and post-intervention survey}

The survey was undertaken entirely online. A preintervention survey was emailed to all participants prior to watching the video, along with a link to the video and electronic copies of the resources. This was followed by a postintervention survey 10-12 weeks later (Table 2). The survey assessed baseline knowledge and attitudes towards current sleep management (pre-intervention survey) and then measured any changes that had taken place in the knowledge and attitudes of individuals over the intervention period (postintervention survey). These outcomes were relevant in assessing whether the interventions had an impact on staff knowledge and behaviours, even if this impact was not enough to change prescribing and administration rates on the wards.

\section{Participants}

The package of educational interventions was designed by a stakeholder group consisting of hospital pharmacists $(n=8)$, primary care pharmacists $(n=4)$, a neurologist with specialist interest in sleep $(n=1)$ and consultant psychiatrists $(n=3)$. Stakeholders were recruited through local networks and selected for their knowledge and experience in insomnia, hypnotics or local practice. A patient and public involvement group was also consulted to consider which messages they would want to ensure were communicated to healthcare staff caring for them. Extensive consultation in the form of draft videos and resources shared via email ensured that these interventions would be generalisable across all care settings. A draft video was also shown at a regional pharmacy conference and feedback was collected on the content and quality, to be integrated into the final version.

The resulting interventions were introduced on in-patient wards $(n=7)$ in one hospital and compared against a control ward. A geographically distant hospital within the same trust was selected for the control ward, to mitigate the risk of migration of practice if staff crosscovered pilot and control wards.

\begin{tabular}{|c|c|}
\hline Pre-intervention survey & Post-intervention survey \\
\hline 1. Please indicate your job role & 1. Please indicate your job role \\
\hline $\begin{array}{l}\text { 2. How confident do you feel } \\
\text { using mediation to help patients } \\
\text { sleep }(/ 5)\end{array}$ & $\begin{array}{l}\text { 2. How confident do you now feel } \\
\text { using mediation to help patients } \\
\text { sleep }(/ 5)\end{array}$ \\
\hline $\begin{array}{l}\text { 3. Please rank the following } \\
\text { sleeping tablets from } 1 \text { 'most } \\
\text { commonly used' to } 5 \text { 'least } \\
\text { commonly used': }\end{array}$ & $\begin{array}{l}\text { 3. Please rank the following } \\
\text { sleeping tablets from } 1 \text { 'should be } \\
\text { most commonly used' to } 5 \\
\text { 'should be least commonly used': }\end{array}$ \\
\hline $\begin{array}{l}\text { - Melatonin } \\
\text { - Temazepam } \\
\text { - Zopiclone } \\
\text { - Zolpidem } \\
\text { - Promethazine }\end{array}$ & $\begin{array}{l}\text { - Melatonin } \\
\text { - Temazepam } \\
\text { - Zopiclone } \\
\text { - Zolpidem } \\
\text { - Promethazine }\end{array}$ \\
\hline $\begin{array}{l}\text { 4. Please explain why you have } \\
\text { ranked the above sleeping } \\
\text { tablets in the order you have } \\
\text { listed }\end{array}$ & $\begin{array}{l}\text { 4. Please explain why you have } \\
\text { ranked the above sleeping tablets } \\
\text { in the order you have listed }\end{array}$ \\
\hline $\begin{array}{l}\text { 5. In your own words, please } \\
\text { describe how you would } \\
\text { manage a patient reporting poor } \\
\text { sleep }\end{array}$ & $\begin{array}{l}\text { 5. How confident do you now feel } \\
\text { about using psychological } \\
\text { strategies such as cognitive- } \\
\text { behavioural therapy for insomnia } \\
\text { (CBTi) to help patients sleep? }\end{array}$ \\
\hline $\begin{array}{l}\text { 6. How confident do you } \\
\text { personally feel about using } \\
\text { psychological strategies such as } \\
\text { cognitive-behavioural therapy } \\
\text { for insomnia (CBTi) to help } \\
\text { patients sleep? }\end{array}$ & $\begin{array}{l}\text { 6. Has your approach to } \\
\text { managing a patient who is } \\
\text { reporting poor sleep changed } \\
\text { since before you did this } \\
\text { training? If so, how? }\end{array}$ \\
\hline
\end{tabular}

\section{Ethics}

All participants gave their informed consent for inclusion before they participated in the study. The study was conducted in accordance with the Declaration of Helsinki, and the protocol was approved by the Ethics Committee of Newcastle University Faculty of Medical Sciences. The project was also registered with the research department at Cumbria, Northumberland, Tyne and Wear NHS Foundation Trust.

\section{Standards}

The SQUIRE statement was used as a scaffold in the writing of this paper. ${ }^{49}$

All wards received the same level of resources for implementation of the intervention. Variation in levels of staff engagement with the interventions may have led to variation between wards, but the interventions themselves were standardised across all pilot wards. The control (comparator) ward had no interventions delivered, but staff were informed that data would be collected to allow for comparison, and interventions made available after the evaluation phase had been completed.

\section{Results}

\section{Hypnotic prescribing and administration rates}

A decrease in the number of administrations occurred on all wards except one, which was a psychiatric intensive care 


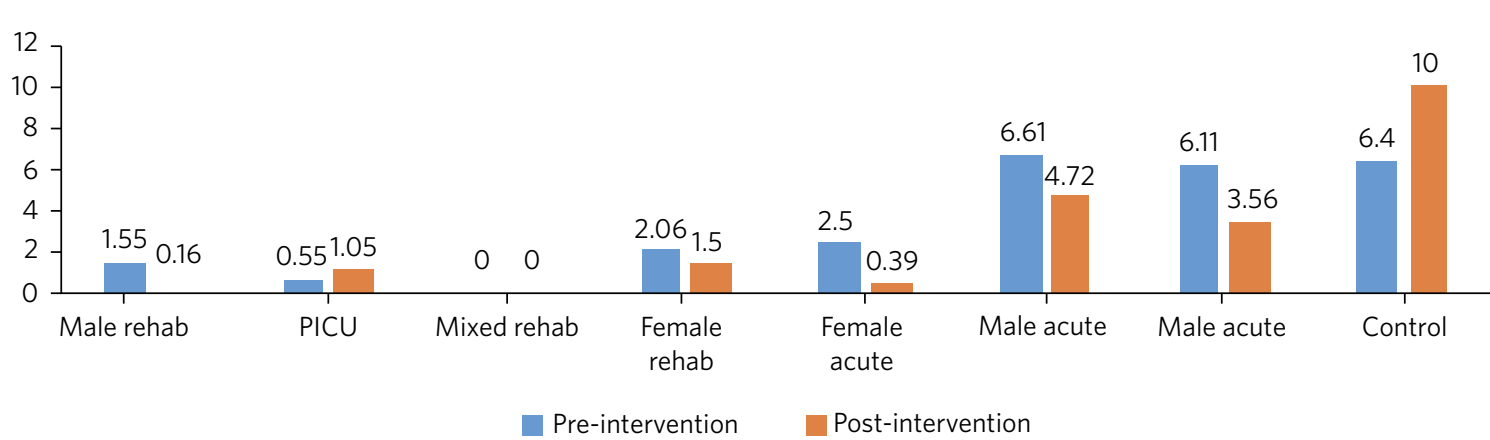

Fig. 2 Average number of recorded administrations of hypnotics per patient pre- and post-educational interventions across eight mental health in-patient wards. Rehab, rehabilitation; PICU, psychiatric intensive care unit.

unit (PICU) treating patients with more challenging behaviours (Fig. 2). Overall, there was a mean reduction of $41 \%$ in the number of hypnotic administrations per patient across the pilot site. This compares with the control site, which showed a $37 \%$ increase in the administrations of hypnotics per patient on the control ward (Table 3).

A decrease in the percentage of patients prescribed hypnotics occurred on $71 \%$ of the pilot wards (Fig. 3). The PICU and female acute wards did not show a decrease, however. Overall, there was a mean reduction of $24 \%$ in the percentage of patients prescribed hypnotics on the pilot site. This compares with the control ward (a mixed-gender rehabilitation ward), which showed no change.

\section{Pre- and post-intervention survey}

A total of 251 staff were invited to participate in the survey. Non-participation reasons included a lack of time, leave of absence and self-perceived adequate pre-existing knowledge in this subject area. Survey response data were collected on the pre-intervention questionnaire $(n=85)$ and postintervention questionnaire $(n=45)$ (total $n=131$ ). Total respondents for both surveys comprised: nurses (preintervention $n=27$; post-intervention $n=13$ ); junior doctors (pre, 7; post, 1); specialty trainees (pre, 6; post, 3); consultants (pre, 3 ; post, 2); pharmacists (pre, 12; post, 12); pharmacy technicians (pre, 6; post, 4); psychologists (pre, 7; post, 2); and 'other' (pre, 17; post, 8). Two respondents did not complete the question about job role.

Before and after the intervention, respondents were asked to rate their confidence (on a Likert scale from 1 , 'not confident' to 5 , 'extremely confident') in using medication versus psychological strategies to help patients sleep. An increase was seen in confidence scores for using medication and for using psychological strategies to help patients sleep (Table 4).

The intervention prompted a change in the order in which hypnotics were considered (Table 5) for sleep management. Melatonin rose in ranking, whereas promethazine fell.

When asked pre-intervention to comment on their rationale behind the ranking decisions they had made, $87 \%$ of respondents mentioned that their ranking of the listed medications was based on what they had 'seen in practice' or 'heard of the most', i.e. choices were being informed by existing practice on the wards that staff worked. Only $9 \%$ of respondents mentioned anything about knowledge of drugs in their response, and no respondents made reference to the evidence base, cost-effectiveness or risk-benefit. For example,

'I picked the medication that I had heard of used within the service I work. Patients are more frequently prescribed promethazine and zopiclone, promethazine is usually offered first. Melatonin I believe is non formulary so is used less regularly. Temazepam is used rarely. I have never heard of zolpidem before.' (Foundation Year 1 doctor)

Asked the same question post-intervention, only $13 \%$ of respondents still mentioned in their responses that their choices were being informed by experience of practice, whereas $76 \%$ mentioned the evidence base, cost-effectiveness and/or risk-benefit in their responses. For example,

'Melatonin is licensed in my client group for insomnia Evidence base supports the use of Z-drugs and benzodiazepines for short term use in insomnia and Z-drugs have a slightly more favourable side-effect profile in terms of longterm dependency. There is little evidence to support the use of sedating antihistamines for insomnia.' (Advanced Pharmacist Practitioner)

\section{Discussion}

\section{Hypnotic prescribing and administration rates}

Among staff working on the in-patient units studied, knowledge about the mechanism of action of hypnotic medications and different sleep disorders was limited and informed by previous practice, not the evidence base. After a short educational video about sleep disorders and hypnotics, the hypnotic prescribing rates decreased by $24 \%$ and the administration rates fell by $41 \%$ compared with the control ward. There was also a difference in which medications were used as hypnotics, with promethazine use being reduced. Staff described a better understanding of sleep and the different available therapies for sleep disorders after the intervention.

An increase in hypnotics issued was seen on the PICU despite the implementation of the educational interventions (Table 2). This may be due to the nature of the patients on this ward, who are more likely to have challenging behaviours and disturbed sleep and to not engage as much with 


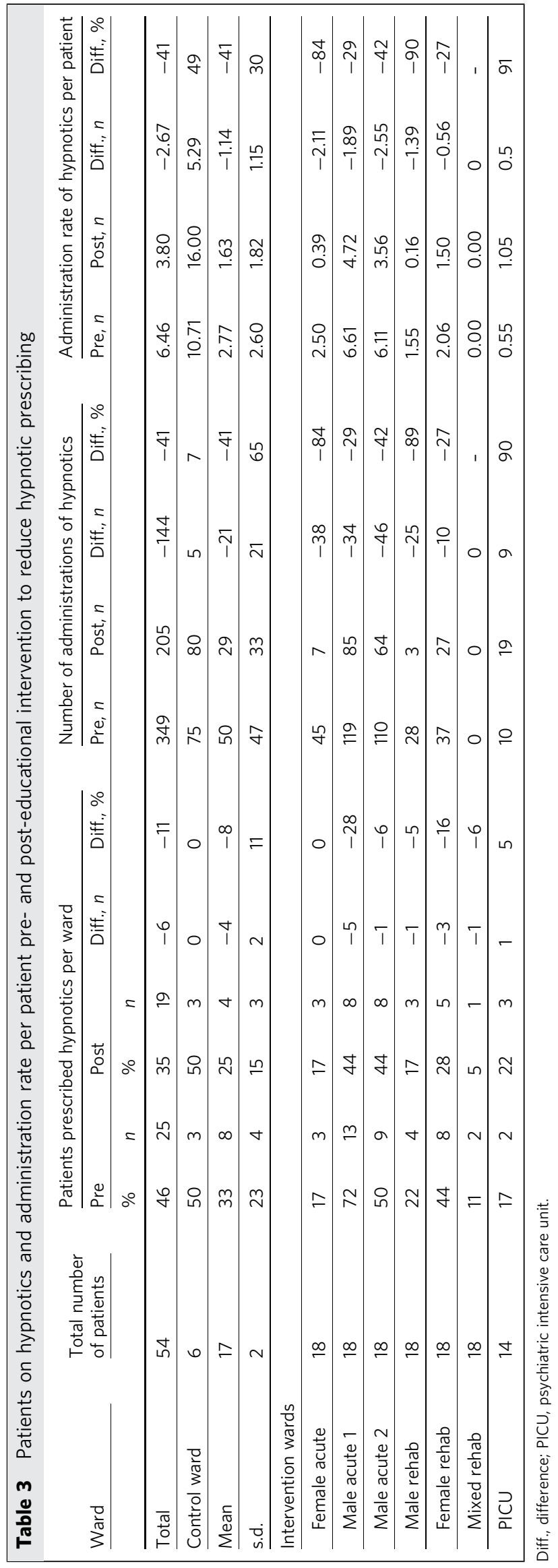

psychological therapies compared with patients admitted to acute and rehabilitation wards.

\section{Pre- and post-intervention survey}

Self-rated confidence scores show a larger improvement in the mean score in relation to medication than in the mean score for psychological strategies for improving sleep. Further support and training may be needed to help staff feel confident in psychological management. Alternatively, this might suggest that, owing to the often acute nature of the in-patient setting, hypnotics are deemed to be of greater utility than psychological strategies in the care of patients struggling with sleep and/or anxiety.

The table ranking the order in which hypnotics were considered (Table 5) shows convergence of practice towards the evidence base. Zolpidem is non-formulary in this geographical area, which is likely to be the reason for it being lowest ranked. Promethazine has fallen in ranking, which is likely to be because the educational package highlighted its weak evidence base and high side-effect profile. Melatonin rose in ranking, possibly due to its favourable side-effect profile and the fact that it is an endogenous hormone involved in regulation of the circadian rhythm. It does, however, have a short half-life and so its use in sleep disorders may be limited, ${ }^{50}$ as was highlighted in the educational package.

An increase in the evidence-based language used when rationalising the rank order of hypnotics suggests that this intervention went some way towards closing the evidencepractice gap in management of poor sleep, as end-user feedback illustrates.

\section{End-user feedback}

Doctors found these resources useful for instigating positive change in relation to hypnotic prescribing, reviewing the clinical need and reducing doses where appropriate:

'Patients were on hypnotics for weeks and weeks and weeks and then stopped immediately at discharge. This is bad practice, and leaves a mess for both GPs [general practitioners] and the patients. These interventions created a change in culture so that the mindset for patients prescribed hypnotics is now to reduce them during the in-patient stay wherever appropriate.' (Specialty trainee)

Nursing staff found these resources helpful in offering alternative solutions to sleep disturbances, other than administering hypnotic medication:

'I feel like, after watching the video, we started to encourage patients to use non-pharmacological methods more. This demonstrated that patients were often showing drug seeking behaviours, as they would often then go to sleep without needing hypnotics.' (Ward manager)

The video was viewed favourably by staff and shone a light on a field of practice that is often overlooked:

'Excellent video, extremely important field that is greatly underestimated, but is pivotal in a patient's physical, mental and emotional wellbeing.' (Consultant) 


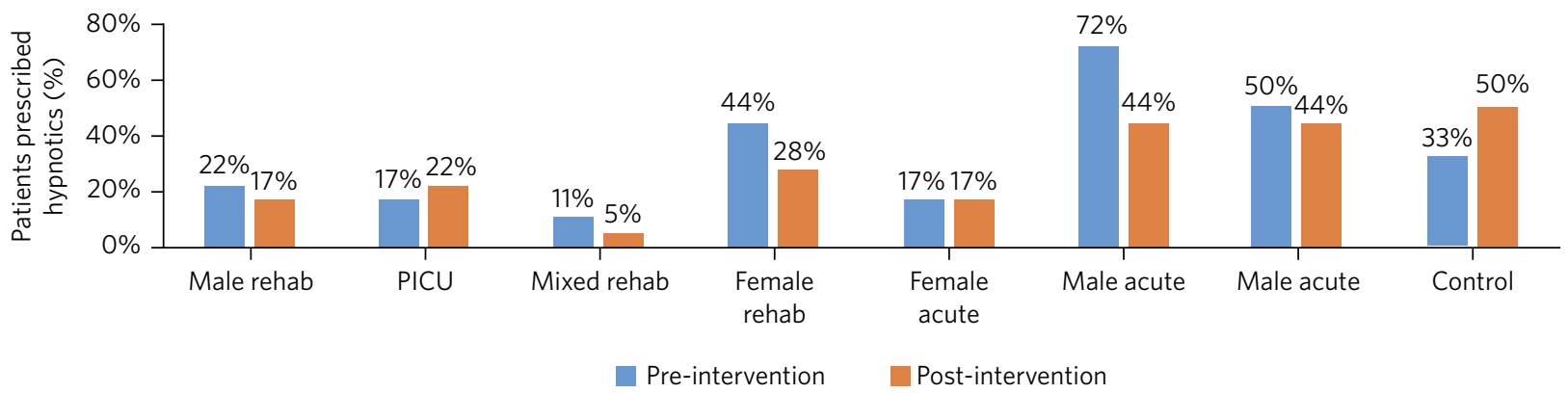

Fig. 3 Percentage of patients prescribed hypnotics per ward pre- and post-educational interventions across eight mental health in-patients wards Rehab, rehabilitation; PICU, psychiatric intensive care unit.

\begin{tabular}{|c|c|c|c|c|}
\hline & Before intervention $(n=86)$ & After intervention $(n=45)$ & Actual difference & Percentage difference \\
\hline \multicolumn{5}{|c|}{ Item 'Using medication to help patients sleep' } \\
\hline Mean score & 2.77 & 3.71 & +0.94 & $+18.8 \%$ \\
\hline \multicolumn{5}{|c|}{ Item 'Using psychological strategies to help patients sleep' } \\
\hline Mean score & 2.40 & 3.18 & +0.78 & $+15.6 \%$ \\
\hline
\end{tabular}

a. Rated on a scale of $0-5$, where 0 indicates no confidence and 5 indicates very confident.

\section{Generalisability}

The presence of the pharmacy team on all wards enabled the proliferation of the intervention among other staff groups. Rotation of nursing staff between wards enabled migration of practice to spread engagement with the interventions across wards. Some wards $(n=2)$ were involved in the SleepWell Project, ${ }^{51}$ which may have motivated staff to engage with further work on sleep, but the impact of SleepWell in relation to this study is unclear. The above aspects may not exist in other settings.

\section{Limitations}

There were several limitations to the study. No data were gathered on which of the interventions was felt to be the most effective, as the intervention package was conceptualised as a whole. The evaluation period was short, owing to

\begin{tabular}{|c|c|}
\hline $\begin{array}{r}\text { Table } 5 \text { Average } \\
\text { first chc } \\
\text { tional ir }\end{array}$ & $\begin{array}{l}\text { order of hypnotics for prescribing, } \\
\text { last choice, before and after educa- } \\
\text { ntion }\end{array}$ \\
\hline $\begin{array}{l}\text { Before intervention } \\
(n=86)\end{array}$ & After intervention $(n=45)$ \\
\hline 1 Zopiclone & 1 Zopiclone (no change) \\
\hline 2 Promethazine & 2 Melatonin (more favourable choice) \\
\hline 3 Temazepam & 3 Temazepam (no change) \\
\hline 4 Melatonin & 4 Promethazine (less favourable choice) \\
\hline 5 Zolpidem & 5 Zolpidem (no change) \\
\hline
\end{tabular}

the limited time span available within the context of the funding. There were fewer responses to the postintervention survey than to the pre-intervention survey, as pre-intervention responses were gathered immediately before accessing the intervention. Post-intervention responses were gathered 10-12 weeks later, to allow time for a change in practice to occur. A shorter interval may have resulted in a lower attrition rate, but seen a smaller change in prescribing and administration rates. Completing the post-intervention survey straight after the resources were accessed may have increased responses but would have allowed less time for reflection. Introducing a prize raffle for completion of the post-intervention survey may also have increased responses.

The control ward was selected because of its geographical distance from the intervention wards to prevent migration of practice. During the course of this study the ward was planned for closure, so admitted fewer and fewer patients, leading to a lower number of patients in this group.

\section{Future work}

Future work could examine the nuances of uptake and benefit between interventions. It might also be valuable to evaluate whether the reduction in inappropriate hypnotic prescriptions is sustained over time. If it is not sustained, measuring how long this change lasts and investigating barriers and enablers for maintaining a change in evidencebased practice would be worthy of future investigation. Further work could also look at the feasibility and uptake of these interventions in primary care and examine barriers and enablers to the delivery of online educational interventions in this setting. 


\section{About the authors}

Alastair Paterson, Pharmacy Department, Cumbria, Northumberland, Tyne and Wear NHS Foundation Trust; and School of Pharmacy, Faculty of Medical Sciences, Newcastle University, Newcastle upon Tyne, UK. Martina Khundakar, Pharmacy Department, Cumbria, Northumberland, Tyne and Wear NHS Foundation Trust; and School of Pharmacy, Faculty of Medical Sciences, Newcastle University, Newcastle upon Tyne, UK Anthony Young, Pharmacy Department, Cumbria, Northumberland, Tyne and Wear NHS Foundation Trust, Newcastle upon Tyne; and School of Pharmacy, Faculty of Health Sciences and Wellbeing, University of Sunderland, UK. Jonathan Ling, Faculty of Health Sciences and Wellbeing, University of Sunderland, UK. Samantha Chakraborty, Department of General Practice, Monash University, Melbourne, Australia. Adam Pattison Rathbone, School of Pharmacy, Faculty of Medical Sciences, Newcastle University; and Newcastle upon Tyne Hospitals NHS Foundation Trust, Newcastle upon Tyne, UK. Stuart Watson, Translational and Clinica Research Institute, Newcastle University; and Inpatient Services, Cumbria Northumberland, Tyne and Wear NHS Foundation Trust, Newcastle upon Tyne, UK. Tim Donaldson, Pharmacy Department, Cumbria, Northumberland, Tyne and Wear NHS Foundation Trust, Newcastle upon Tyne, UK. Kirstie N Anderson, Department of Neurology, Faculty of Medical Sciences, Newcastle University, Newcastle upon Tyne; and Regional Sleep Disorders Service, Newcastle upon Tyne Hospitals NHS Foundation Trust, UK

\section{Data availability}

The data that support the findings of this study are available from the corresponding author, A.P., upon reasonable request.

\section{Acknowledgements}

A.P. thanks the Academic Health Science Network for the North East and North Cumbria (AHSN NENC) for the funding of this project; the pharmacy department, Cumbria, Northumberland, Tyne and Wear NHS Foundation Trust, for their support in releasing staff time to conduct this work; the Newcastle University VOICE patient and public involvement group for their advice in this project; and the Regional Sleep Service, Newcastle upon Tyne Hospitals, for arranging for the professional production of the video resource.

\section{Author contributions}

Conceptualisation: A.P., M.K., A.Y., A.P.R., S.W. and K.N.A.; data curation: A.P.; formal analysis, A.Y.; funding acquisition: A.P., S.W., T.D. and K.N.A.; Methodology: A.P., A.Y., J.L., S.C. and K.N.A.; project administration, A.P.; resources, A.P.R. and K.N.A.; supervision, M.K., J.L., S.C., A.P.R., S.W., T.D. and K.N.A.; writing - original draft, A.P. and K.N.A.; writing - review and editing, M.K., A.Y., J.L., S.C., A.P.R., S.W., T.D. and K.N.A.

\section{Funding}

This work was supported by the Academic Health Science Network for the North East and North Cumbria (AHSN NENC), project number MO3. The funders had no role in the design of the study; in the collection, analyses, or interpretation of data; in the writing of the manuscript; or in the decision to publish the results.

\section{Declaration of interest}

None.

\section{References}

1 Riemann D, Berger M, Voderholzer U. Sleep and depression - results from psychobiological studies: an overview. Biol Psychol 2001; 57 67-103.
2 Harvey AG. Sleep and circadian rhythms in bipolar disorder: seeking synchrony, harmony, and regulation. Am J Psychiatry 2008; 165: 820-9.

3 Sarkar S, Katshu MZUH, Nizamie SH, Praharaj SK. Slow wave sleep deficits as a trait marker in patients with schizophrenia. Schizophr Res 2010; 124: 127-33.

4 Cohen S, Conduit R, Lockley SW, Rajaratnam SM, Cornish KM. The relationship between sleep and behavior in autism spectrum disorder (ASD): a review. J Neurodev Disord 2014; 6: 44

5 Owens JA. The ADHD and sleep conundrum: a review. J Dev Behav Pediatr 2005; 26: 312-22.

6 Brower KJ, Perron BE. Sleep disturbance as a universal risk factor for relapse in addictions to psychoactive substances. Med Hypotheses 2010; 74: 928-33.

7 Gopalakrishnan A, Ji LL, Cirelli C. Sleep deprivation and cellular responses to oxidative stress. Sleep 2004; 27 : 27-35.

8 Grandner MA, Jackson NJ, Pak VM, Gehrman PR. Sleep disturbance is associated with cardiovascular and metabolic disorders. J Sleep Res 2012; 21: 427-33.

9 Soldatos CR. Insomnia in relation to depression and anxiety: epidemiologic considerations. J Psychosom Res 1994; 38: 3-8.

10 Walker RL, Talavera DC, Nomamiukor F, Madubata IJ, Alfano C, Vujanovic AA. Sleep-related problems and suicide behavior and ideation among Black and White trauma-exposed psychiatric inpatients. Compr Psychiatry 2019; 91: 22-8.

11 Ribeiro JD, Pease JL, Gutierrez PM, Silva C, Bernert RA, Rudd MD, et al. Sleep problems outperform depression and hopelessness as cross sectional and longitudinal predictors of suicidal ideation and behavior in young adults in the military. J Affect Disord 2012; 136: 743-50.

12 Doğan O, Ertekin S, Doğan S. Sleep quality in hospitalized patients. J Clin Nurs 2005; 14: 107-13.

13 Talih F, Ajaltouni J, Ghandour H, Abu-Mohammad AS, Kobeissy F. Insomnia in hospitalized psychiatric patients: prevalence and associated factors. Neuropsychiatr Dis Treat 2018; 14: 969-75.

14 Koffel E, Thuras P, Chakravorty S, Germain A, Khawaja IS. Poor sleep quality at discharge as a predictor of readmission to a psychiatry partial hospitalization program. Prim Care Companion CNS Disord 2015; 17(6): 10.4088/PCC.15I01826

15 Nedeltcheva AV, Scheer FAJL. Metabolic effects of sleep disruption, links to obesity and diabetes. Curr Opin Endocrinol Diabetes Obes 2014; 21: $293-8$

16 Parish JM, Shepard JW. Cardiovascular effects of sleep disorders. Chest 1990; 97: 1220-6.

17 Partinen $M$, Jamieson A, Guilleminault C. Long-term outcome for obstructive sleep apnea syndrome patients: mortality. Chest 1988; 94 1200-4

18 Thorpy M. International classification of sleep disorders. In Sleep Disorders Medicine: Basic Science, Technical Considerations and Clinical Aspects (ed S Chokroverty): 475-84. Springer, 2017.

19 Perry GS, Patil SP, Presley-Cantrell LR. Raising awareness of sleep as a healthy behavior. Prev Chronic Dis 2013; 10: E133.

20 Janhsen $\mathrm{K}$, Roser $\mathrm{P}$, Hoffmann $\mathrm{K}$. The problems of long-term treatment with benzodiazepines and related substances. Dtsch Arztebl Int 2015; 112: 1-7.

21 Ford DE, Kamerow DB. Epidemiologic study of sleep disturbances and psychiatric disorders: an opportunity for prevention? JAMA 1989; 262: $1479-84$

22 Romiszewski S, May FEK, Homan EJ, Norris B, Miller MA, Zeman A Medical student education in sleep and its disorders is still meagre 20 years on: a cross-sectional survey of UK undergraduate medical education. J Sleep Res 2020; 29(6): e12980.

23 O'Regan D. The misprescribing of Z-drugs for insomnia. ACNR 2019; 18 (3): $10-12$.

24 Hallahan BP, Murray IT, McDonald C. Benzodiazepine and hypnotic prescribing in an acute adult psychiatric in-patient unit. Psychiatr Bull 2009; 33: 12-4 
25 Guina J, Merrill B. Benzodiazepines II: waking up on sedatives: providing optimal care when inheriting benzodiazepine prescriptions in transfer patients. J Clin Med 2018; 7(2): 20

26 Ancoli-Israel S, Richardson GS, Mangano RM, Jenkins L, Hall P, Jones WS. Long-term use of sedative hypnotics in older patients with insomnia. Sleep Med 2005; 6: 107-13.

27 Li C-T, Bai Y-M, Lee Y-C, Mao W-C, Chen M-H, Tu P-C, et al. High dosage of hypnotics predicts subsequent sleep-related breathing disorders and is associated with worse outcomes for depression. Sleep 2014; 37: 803-9.

28 Baldwin DS, Aitchison K, Bateson A, Curran HV, Davies S, Leonard B, et al. Benzodiazepines: risks and benefits. A reconsideration. J Psychopharmacol 2013; 27: 967-71.

29 Weymann D, Gladstone EJ, Smolina K, Morgan SG. Long-term sedative use among community-dwelling adults: a population-based analysis. CMAJ Open 2017; 5: E52-60.

30 Davies J, Rae TC, Montagu L. Long-term benzodiazepine and Z-drugs use in England: a survey of general practice. Br J Gen Pract 2017; 67: e609-13.

31 Wilson S, Anderson K, Baldwin D, Dijk D-J, Espie A, Espie C, et al. British Association for Psychopharmacology consensus statement on evidence-based treatment of insomnia, parasomnias and circadian rhythm disorders: an update. J Psychopharmacol 2019; 33: 923-47.

32 Reeve E, Gnjidic D, Long J, Hilmer S. A systematic review of the emerging definition of 'deprescribing' with network analysis: implications for future research and clinical practice. $\mathrm{Br} J$ Clin Pharmacol 2015; 80: 1254-68.

33 Ng BJ, Le Couteur DG, Hilmer SN. Deprescribing benzodiazepines in older patients: impact of interventions targeting physicians, pharmacists, and patients. Drugs Aging 2018; 35: 493-521.

34 Kuntz J, Kouch L, Christian D, Peterson PL, Gruss I. Barriers and facilitators to the deprescribing of nonbenzodiazepine sedative medications among older adults. Perm J 2018; 22: 17-157.

35 Guina J, Merrill B. Benzodiazepines I: upping the care on downers: the evidence of risks, benefits and alternatives. J Clin Med 2018; 7(2): 17.

36 Moloney ME. 'Sometimes, it's easier to write the prescription': physician and patient accounts of the reluctant medicalisation of sleeplessness. Sociol Health IIIn 2017; 39: 333-48.

37 Stores G. Multifactorial influences, including comorbidities, contributing to sleep disturbance in children with a neurodevelopmental disorder. CNS Neurosci Ther 2016; 22: 875-9.

38 Sirdifield C, Anthierens S, Creupelandt H, Chipchase SY, Christiaens T, Siriwardena AN. General practitioners' experiences and perceptions of benzodiazepine prescribing: systematic review and meta-synthesis. BMC Fam Pract 2013; 14: 191
39 Wallis L. Barriers to implementing evidence-based practice remain high for U.S. nurses. Am J Nurs 2012; 112(12): 15.

40 Brower KJ. Assessment and treatment of insomnia in adult patients with alcohol use disorders. Alcohol 2015; 49: 417-27.

41 Horne S, Hay K, Watson S, Anderson KN. An evaluation of sleep disturbance on in-patient psychiatric units in the UK. BJPsych Bull 2018; 42. $193-7$.

42 Garcia AN, Salloum IM. Polysomnographic sleep disturbances in nicotine, caffeine, alcohol, cocaine, opioid, and cannabis use: a focused review. Am J Addict 2015; 24: 590-8.

43 Christodulu KV, Durand VM. Reducing bedtime disturbance and night waking using positive bedtime routines and sleep restriction. Focus Autism Dev Disabil 2004; 19: 130-9.

44 Lau R, Stevenson F, Ong BN, Dziedzic K, Treweek S, Eldridge S, et al. Achieving change in primary care-effectiveness of strategies for improving implementation of complex interventions: systematic review of reviews. BMJ Open 2015; 5(12): e009993.

45 Jessup RL, Osborne RH, Buchbinder R, Beauchamp A. Using co-design to develop interventions to address health literacy needs in a hospitalised population. BMC Health Serv Res 2018; 18(1): 989.

46 Graham ID, Logan J, Harrison MB, Straus SE, Tetroe J, Caswell W, et al. Lost in knowledge translation: time for a map? J Contin Educ Health Prof 2006; 26: 13-24.

47 Richardson R, Paterson A, Rathbone A, Blagburn J, Husband A Insomnia: diagnosis and prevention. Pharm J 2020; 304(7938): 10.1211/PJ.2020.20208004

48 Paterson A, Richardson R, Rathbone A, Blagburn J, Husband A Insomnia disorder: management strategies. Pharm J 2020; 305 (7939): 10.1211/PJ.2020.20208111.

49 Pinnock $H$, Barwick $M$, Carpenter CR, Eldridge S, Grandes G, Griffith CJ, et al. Standards for reporting implementation studies (StaRI) statement. BMJ 2017; 356: i6795.

50 Quera-Salva MA, Claustrat B. Mélatonine : aspects physiologiques et pharmacologiques en relation avec le sommeil, intérêt d'une forme galénique à libération prolongée (Circadin ${ }^{\circledR}$ ) dans l'insomnie [Melatonin: Physiological and pharmacological aspects related to sleep: The interest of a prolonged-release formulation (Circadin ${ }^{\circledR}$ ) in insomnia]. L'encephale, 2018, 44: 548-57.

51 Novak C, Packer E, Paterson A, Roshi A, Locke R, Keown P, et al. Feasibility and utility of enhanced sleep management on in-patient psychiatry wards. BJPsych Bull 2020; 44: 255-60.

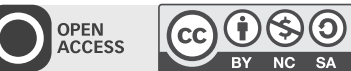

